\title{
Temporal Alteration of Connexin43 Localization during Ultraviolet Light-induced Apoptosis
}

\author{
Wuxiong Zhou ${ }^{1}$, Masahito Oyamada ${ }^{1}$, Yumiko Oyamada ${ }^{1}$ and Tetsuro Takamatsu ${ }^{1}$ \\ ${ }^{1}$ Department of Pathology and Cell Regulation, Kyoto Prefectural University of Medicine, Kyoto 602-8566, Japan
}

Received April 8, 2004; accepted May 10, 2004

\begin{abstract}
Although gap junctions are thought to play an important role in moderating apoptosis, the basic phenomena underlying when and where the alterations of gap junctions occur during apoptosis have not been well documented. In this study we analyzed the spatiotemporal changes of connexin (Cx) during UV light-induced apoptosis using Cx43enhanced green fluorescent protein (EGFP)-expressing HeLa cells, and compared them with those of mitochondrial membrane potential (MMP) using tetramethylrhodamine ethyl ester (TMRE) and nuclear morphological observation using Hoechst 33342. At 2 hr post-UV-irradiation, a third of the cells were TMRE negative, i.e., they showed the loss of MMP, but with slight nuclear fragmentation, and high percentages of linear

Cx43-EGFP plaques were found among both TMRE-positive and TMRE-negative cells. At $4 \mathrm{hr}$ post-UV-irradiation, the percentage of these linear plaques was decreased, and both punctate and diffuse localization of Cx43-EGFP were noted in the cytoplasm of TMRE-negative cells without nuclear fragmentation. At 8 hr post-irradiation, punctate cytoplasmic localization of Cx43-EGFP was noted in TMRE-negative cells with nuclear fragmentation. Treatment with the caspase inhibitor Z-VAD-FMK blocked nuclear fragmentation and partially preserved both gap junctional plaques and MMP. These results indicate that, during apoptosis, Cx mobilization into the cytoplasm occurs after MMP depolarization but before nuclear fragmentation and that this alteration partly depends on caspase.
\end{abstract}

Key words: connexin43, apoptosis, caspase, mitochondrial membrane potential

\section{Introduction}

Gap junctions form intercellular channels and mediate direct transfer of low-molecular-weight (less than about 1 $\mathrm{kD})$ metabolites, ions, and small molecules between neighboring cells. Because some second messengers can be transduced through gap junction channels, gap junctional intercellular communication is considered to play an important role in tissue homeostasis, growth control, and cell differentiation $[4,7,10,20]$. Gap junctional channels are composed of hexagonal arrangements of oligomeric proteins called connexins (Cxs) [2]. Thus far, 20 mouse genes and 21 human genes for connexins have been identified [24].

Apoptosis is an active homeostatic process of cell death found in multicellular organisms that is important for many

Correspondence to: Masahito Oyamada, M.D., Ph.D., Department of Pathology and Cell Regulation, Kyoto Prefectural University of Medicine, Kawaramachi Hirokoji, Kamigyo-ku, Kyoto 602-8566, Japan. E-mail: oyamada@koto.kpu-m.ac.jp biological processes [25]. The apoptotic process involves a series of biochemical steps and structural changes including mitochondrial membrane potential (MMP) depolarization, chromatin condensation, nuclear fragmentation, cytoskeletal reorganization, cell shrinkage, and membrane blebbing [3, 16]. Caspases have been implicated as mediators of apoptosis in a variety of mammalian cell types [11, 18]. For example, after UV-C irradiation, caspase-8, caspase-9 and caspase- 3 are activated in fibroblasts [5]. Caspase- 9 and its downstream caspases are detected in $3 \mathrm{SB}$ and Jurkat cells [23]. HeLa cells and MCF-7 cells also show caspase-9 activation after UV-C irradiation [23].

Controversy exists as to the role of gap junctions and/or connexins in cell death and survival. On the one hand, it has been reported that $\mathrm{Cx} 43$ is capable of transducing cell survival signals in response to extracellular cues [21], and mediates gap junctional intercellular communicationindependent resistance to cell injury [15]. Conversely, other reports showed that cell coupling via gap junctions made of $\mathrm{Cx} 43$ can enhance or propagate cell death in some 
cancer cells $[8,9]$. Thus, the role of gap junctions or $\mathrm{Cx} 43$ in apoptosis has remained uncertain. Moreover, the basic phenomena underlying when and where the alterations of gap junctions occur during apoptosis have not been well documented. In this study, we examined the fate of $\mathrm{Cx} 43$ during apoptosis in order to answer the following questions: (1) Does $\mathrm{Cx} 43$ localization change during apoptosis? (2) If so, when? (3) Does alteration of $\mathrm{Cx} 43$ depend on caspase activation? To answer these questions, we analyzed the spatiotemporal changes of $\mathrm{Cx} 43$ during apoptosis in $\mathrm{Cx} 43$-enhanced green fluorescent protein (EGFP)-expressing HeLa cells by confocal microscopy and conventional fluorescence microscopy, and observed the time course of alteration of $\mathrm{Cx} 43$ and depolarization of MMP and nuclear fragmentation. In addition, we examined the effect of a broad-range caspase inhibitor, Z-VAD-FMK, on apoptosis progression and alternation of $\mathrm{Cx} 43$ localization.

\section{Materials and Methods}

\section{Cell culture}

The establishment of Cx43-EGFP-expressing HeLa cell lines and the formation of functional gap junctions made of Cx43-EGFP in these cell lines were described in our previous paper [19]. For experiments, aliquots of cells $\left(1 \times 10^{5}\right)$ were plated on $35-\mathrm{mm}$ dishes and cultured for 4 days in Dulbecco's modified Eagle's medium (DMEM) (Nissui Pharmaceutical) containing $10 \%$ heat-inactivated fetal bovine serum (FBS) (Gibco), 2 mM L-glutamine, $100 \mathrm{U} / \mathrm{ml}$ penicillin, and $100 \mu \mathrm{g} / \mathrm{ml}$ streptomycin (Gibco) at $37^{\circ} \mathrm{C}$ in a humidified incubator with $5 \% \mathrm{CO}_{2}$ and $95 \%$ air. An examination showed that $\mathrm{Cx} 43$-EGFP plaques were well formed after 4 days' culture.

\section{Induction of apoptosis by UV irradiation}

Cells were washed twice with phosphate-buffered saline (PBS), placed in a small amount of PBS $(200 \mu \mathrm{L}$ in a $35-\mathrm{mm}$ culture dish), and exposed to UV rays (UV-C; wavelength, $254 \mathrm{~nm}$ ) at an energy dose of $180 \mathrm{~mJ} / \mathrm{cm}^{2}$ by UV crosslinker (UV Stratalinker 2400, Stratagene), according to a method described by Goldstein et al. [6]. After UV irradiation, PBS was aspirated and the cell culture medium was added. Post-irradiation incubation was continued at $37^{\circ} \mathrm{C}$ in a humidified incubator with $5 \% \mathrm{CO}_{2}$ and $95 \%$ air.

\section{Cell morphology observation by time-lapse phase-contrast microscopy}

To analyze the effect of the UV irradiation on cell morphology, 35-mm dishes with 2-mm grid were used for culture. Before UV irradiation and after various periods of post-irradiation incubation, cells were observed with a phase-contrast microscope (Olympus IMT-2, equipped with an NEC TI-23A CCD camera). Images were taken at the same field.

\section{Measurement of apoptosis frequency}

To measure the frequency of apoptosis induced by UV-
$\mathrm{C}$, at 2, 4, 8, 12, and $24 \mathrm{hr}$ after UV irradiation, detached cells in culture dishes were collected into small tubes. The undetached cells were treated with $0.05 \%$ trypsin-EDTA and harvested into the same tubes. Cell pellets were washed in PBS, fixed with $4 \%$ paraformaldehyde for $10 \mathrm{~min}$, and stained with $10 \mu \mathrm{g} / \mathrm{mL}$ Hoechst 33342 (Molecular Probes) for $30 \mathrm{~min}$ at room temperature. The cell suspensions in PBS with Hoechst dye were dropped onto glass slides, covered with coverslips, and observed under a conventional fluorescence microscope (Olympus AX 70) which was equipped with a cooled CCD camera (Quantix photometrics) and IPLab software (Scanalytics, Inc.). For each sample, at least 5 images were taken randomly. More than 1000 cells at each time-point were counted. The percentages of apoptotic cells characterized by nuclear fragmentation were calculated. Experiments were performed for three dishes for each time-point.

\section{Time-lapse fluorescence microscopy of CX43-EGFP localization after $\boldsymbol{U}$ V irradiation}

To observe the localization of Cx43-EGFP during apoptosis, a time-lapse fluorescence microscopy experiment was carried out. Before UV irradiation, cells were stained with Hoechst 33342 for $30 \mathrm{~min}$. After UV irradiation, a $\mathrm{CO}_{2}$ independent culture medium (Gibco), supplemented with $10 \%$ FBS, $2 \mathrm{mM}$ L-glutamine, $100 \mathrm{U} / \mathrm{mL}$ penicillin, and 100 $\mu \mathrm{g} / \mathrm{mL}$ streptomycin, was used for time-lapse observation. Cells were incubated at $37^{\circ} \mathrm{C}$ in an air incubator after UV irradiation and observed at room temperature at various timepoints under a fluorescence microscope (Olympus AX 70) equipped with a water objective (Olympus LUMPlanFl 60x/ $0.90 \mathrm{~W}$ ) and a cooled CCD camera. Images were taken at the same field using IPLab software. The exposure time was fixed manually for $1 \mathrm{sec}$. After observation and taking of pictures at each time-point, the culture medium was changed to fresh medium and the culture dish was returned to the $37^{\circ} \mathrm{C}$ air incubator and cultured until the next time-point.

To compare the time-courses of MMP depolarization, the alterations of Cx43-EGFP, and morphological changes of nuclei, a random area observational study by fluorescence microscopy was performed. Cells were first stained with Hoechst 33342. At 2, 4, $8 \mathrm{hr}$ after UV irradiation, the cells were stained with $40 \mathrm{nM}$ tetramethylrhodamine ethyl ester (TMRE) (Molecular Probes) for 20 min under culture condition. Pictures were taken from different dishes immediately after TMRE staining at 2, 4 and $8 \mathrm{hr}$ post-irradiation.

\section{Confocal laser scanning microscopy of CX43-EGFP and TMRE after $U$ V irradiation}

At 2, 4, $8 \mathrm{hr}$ after UV irradiation, the cells were stained with TMRE and observed with a confocal laser scanning microscope (FluoView, Olympus). An argon-krypton laser produced excitation bands at $488 \mathrm{~nm}$ for Cx43-EGFP, bands at $568 \mathrm{~nm}$ for TMRE, and monochromatic light for transmitted images. Confocal images were taken by using a phase-contrast objective lens $(40 \times$, N.A. 0.60 LCPIanFl, Olympus) and two-time zooming. The alterations of $\mathrm{Cx} 43-$ 
EGFP localization and MMP were compared.

\section{Inhibition of apoptosis by treatment with a caspase inhibitor}

A cell-permeable, irreversible tripeptide inhibitor of caspase, Z-Val-Ala-Asp-FMK, non-omethylated (Z-VADFMK) (ICN Pharmaceuticals, Inc.) was used. One hundred micromoles of Z-VAD-FMK (final concentration) in culture medium was added immediately after UV irradiation, and after $1 \mathrm{hr}$, it was washed out and replaced with culture medium. Cells were cultured until various time-points and stained with Hoechst 33342 and TMRE, and then observed by fluorescence microscopy and confocal laser scanning microscopy as described above.

\section{Morphometric analysis}

To estimate the time course of MMP depolarization and alteration of $\mathrm{Cx} 43-\mathrm{EGFP}$, we performed morphometric analysis of the fluorescence intensity of TMRE on the cells with linear Cx43-EGFP plaques before and $2 \mathrm{hr}$ after UV irradiation and calculated the percentage of cells with positive and negative TMRE staining. Confocal images for TMRE and Cx43-EGFP were collected by adjusting the sensitivity of the photomultiplier so that the intensity of TMRE and Cx43-EGFP fluorescence in the untreated control cells was below the 4095 level. The conditions for taking images were standardized as following: laser power, $35 \mathrm{~mW}$; ND filter, 50\%; photomultiplier voltage for Cx43-EGFP, $-680 \mathrm{~V}$; photomultiplier voltage for TMRE, $-740 \mathrm{~V}$. In this study, the Cx43-EGFP plaques with lengths of more than $5 \mu \mathrm{m}$ and of linear morphology were defined as linear plaques. The fluorescence intensity of TMRE on single cells with Cx43-EGFP linear plaques was analyzed by FluoView software (Olympus). The cells with TMRE fluorescence intensity between 2000 and 4000 (arbitrary units) were counted as TMRE $(++)$ cells; those with fluorescence intensity between 1000 and 2000 (arbitrary units) were counted as TMRE (+) cells to distinguish the decrement of TMRE fluorescence. Cells with fluorescence intensity less than 1000 (arbitrary units) were counted as TMRE (-) cells to indicate the loss of MMP. At this intensity level, the red fluorescence of TMRE was very dark or could not be seen on the images. At two hours after UV irradiation, a total of 106 cells with $53 \mathrm{Cx} 43$-EGFP linear plaques from 10 pictures were analyzed.

For morphometric analysis of the effect of the caspase inhibitor on MMP depolarization and Cx43-EGFP localization after UV irradiation, ten images at each time-point were taken with the confocal laser scanning microscope. Cells that lost MMP showed negative TMRE staining. The percentage of TMRE-negative cells after UV irradiation with or without Z-VAD-FMK treatment was measured. To analyze the effect of Z-VAD-FMK on Cx43-EGFP, ten images were taken at the areas where Cx43-EGFP was distributed at the time-point before and at 2, 4, and $8 \mathrm{hr}$ after UV irradiation with or without treatment with Z-VAD-FMK. In 10 pictures, the total number of cells with Cx43-EGFP, regardless of their localization or morphology, and the total number of cells with linear plaques were counted. The percentage of cells with linear plaques was calculated as the number of cells with linear plaques divided by that of the number of cells with Cx43-EGFP.

\section{Results}

\section{UV induced morphological changes and apoptosis in CX43-EGFP expressing HeLa cells}

UV irradiation caused various morphologic alterations with the extension of post-irradiation incubation time (Fig. 1). As Figure 1B demonstrates, some cells began to display rounded shape at $2 \mathrm{hr}$ after UV irradiation. Cell rounding was more pronounced at 4 to $12 \mathrm{hr}$ (Fig. 1C-E). Many cells were detached and floated in the culture medium at $24 \mathrm{hr}$ after UV irradiation (Fig. 1F).

Morphological changes of nuclei were detected by Hoechst 33342 staining and fluorescence microscopy. As shown in Figure 2, condensed chromatin and fragmented nuclei became more pronounced with time after UV irradiation (Fig. 2A-F). The percentages (mean \pm S.D.) of apoptotic cells characterized by nuclear fragmentation were $5.4 \% \pm 1.2 \%$, $21.2 \% \pm 5.6 \%$, and $33.6 \% \pm 6.2 \%$ at 2,4 , and $8 \mathrm{hr}$, respectively. The percentage increased to $42.6 \% \pm 8.7 \%$ at $12 \mathrm{hr}$ and reached $65.3 \% \pm 8.0 \%$ at $24 \mathrm{hr}$ (Fig. $2 \mathrm{G}$ ).

\section{Cx43-EGFP translocated into the cytoplasm after MMP depolarization and before nuclear fragmentation}

Cx43-EGFP localization and nuclear morphology were assessed by conventional fluorescence time-lapse microscopy. Images of Cx43-EGFP and nuclei before and after UV irradiation are presented in Figure 3. Before UV irradiation, Cx43-EGFP exhibited linear and/or punctate fluorescence between adjacent cells (Fig. 3A). In contrast, at $4 \mathrm{hr}$ after irradiation, the strands of $\mathrm{Cx} 43$-EGFP linear plaques were disrupted, and only small pieces of punctate Cx43-EGFP were observed (Fig. 3B). At $8 \mathrm{hr}$, the number of punctate Cx43-EGFP was decreased, and most of the Cx43-EGFP was degraded (Fig. 3C). The alteration of Cx43-EGFP distribution appeared to occur before nuclear fragmentation. In the time-lapse observation for $8 \mathrm{hr}$, the alteration of Cx43-EGFP was noted; however, only a few cells showed nuclear fragmentation. In negative control experiments without UV irradiation, Cx43-EGFP linear plaques were preserved in the time-lapse observation for $8 \mathrm{hr}$ (data not shown).

To examine the time course of $\mathrm{Cx} 43$-EGFP alteration during the progression of apoptosis, especially in its relationship to MMP depolarization, we stained the cells with TMRE to evaluate MMP. As shown in Figure 4, Cx43EGFP linear plaques were found at appositional membranes between neighboring cells in the control without UV irradiation, and all cells showed positive staining for TMRE (Fig. 4A). Two hours after UV irradiation, many cells had lost their MMP and in most cells the TMRE fluorescence intensity was decreased. Cx43-EGFP linear plaques were observed at the border of adjacent cells that showed negative 

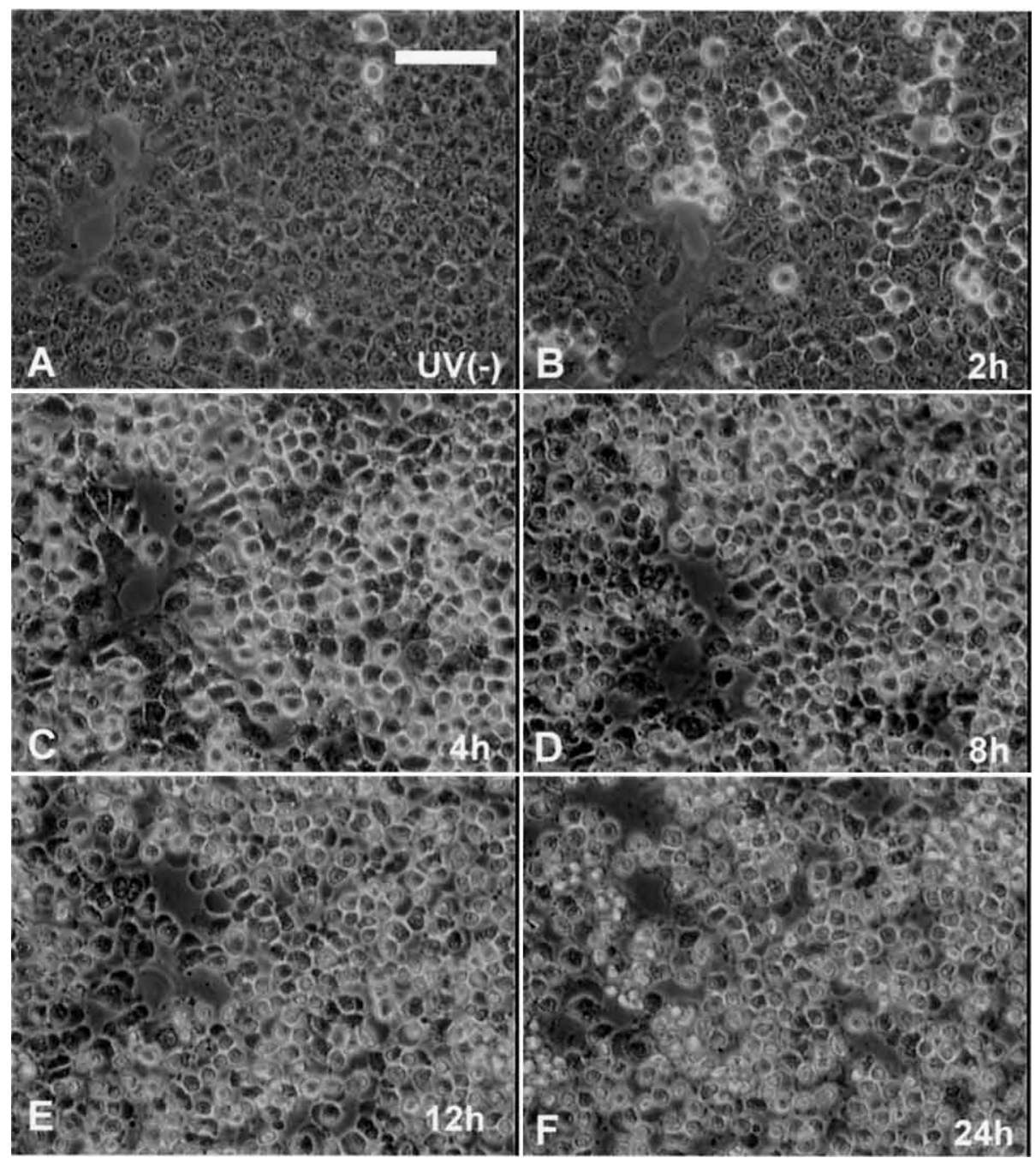

Fig. 1. Phase-contrast photomicrographs of cultured Cx43-EGFP-expressing HeLa cells after UV-C irradiation at the dose of $180 \mathrm{~mJ} / \mathrm{cm}^{2}$. Panel A was taken before UV irradiation; B, C, D, E and $\mathbf{F}$ are pictures taken at the same field at 2, 4, 8, 12 and $24 \mathrm{hr}$ after UV exposure, respectively. At the beginning of the experiment, cells are confluent and flat (A). With the extension of post-irradiation culture time, many cells become rounded (B-E) and detach or float in the culture medium $(\mathbf{F})$. Bar $=90 \mu \mathrm{m}$.

staining for TMRE, indicating that the localization of $\mathrm{Cx} 43-$ EGFP was preserved in cells which lacked MMP (Fig. 4B). Quantitative analysis at $2 \mathrm{hr}$ post-irradiation showed that, of the cells with linear plaques $47.2 \%$ were TMRE $(+)$, i.e., had decreased MMP, while 39.6\% were TMRE (-), i.e., had loss of MMP (Fig. 4E). These data suggest that MMP depolarization occurred prior to Cx43-EGFP disorganization. At $4 \mathrm{hr}$, a dramatic loss of TMRE fluorescence was noted. Cx43EGFP plaques were disrupted, and small spots of punctate Cx43-EGFP were localized in the cytoplasm. At this period, the number of cells with diffuse localization of $\mathrm{Cx} 43$-EGFP in the cytoplasm was increased (Fig. 4C). Eight hours after UV irradiation, the majority of the cells showed dissipated MMP, and the green fluorescence intensity of Cx43-EGFP was decreased (Fig. 4D).

\section{A caspase inhibitor inhibited apoptosis and postponed the disruption of Cx43-EGFP}

The caspase inhibitor Z-VAD-FMK significantly inhibited apoptosis induced by UV irradiation. As demonstrated in Figure 5, Z-VAD-FMK inhibited MMP depolarization at $2 \mathrm{hr}$ after UV irradiation (Fig. 5E), and it also blocked nuclear fragmentation in the UV-treated cells at $8 \mathrm{hr}$. $\mathrm{Cx} 43-$ EGFP linear plaques were detected between cells at $8 \mathrm{hr}$, although punctate Cx43-EGFP was also present in the cytoplasm (Fig. 5F). Compared to cells without treatment with Z-VAD-FMK, disruption of Cx43-EGFP after UV irradiation was reduced.

Confocal laser scanning microscopy also demonstrated that in the cells treated with UV and Z-VAD-FMK, MMP depolarization was blocked at $2 \mathrm{hr}$ and retarded at 4 and $8 \mathrm{hr}$ (Fig. 6B-D). Morphometric analysis showed that after UV irradiation, the percentages of cells which had lost MMP (TMRE-negative) were $33.7 \% \pm 6.0 \%, 78.1 \% \pm 12.0 \%$, and 

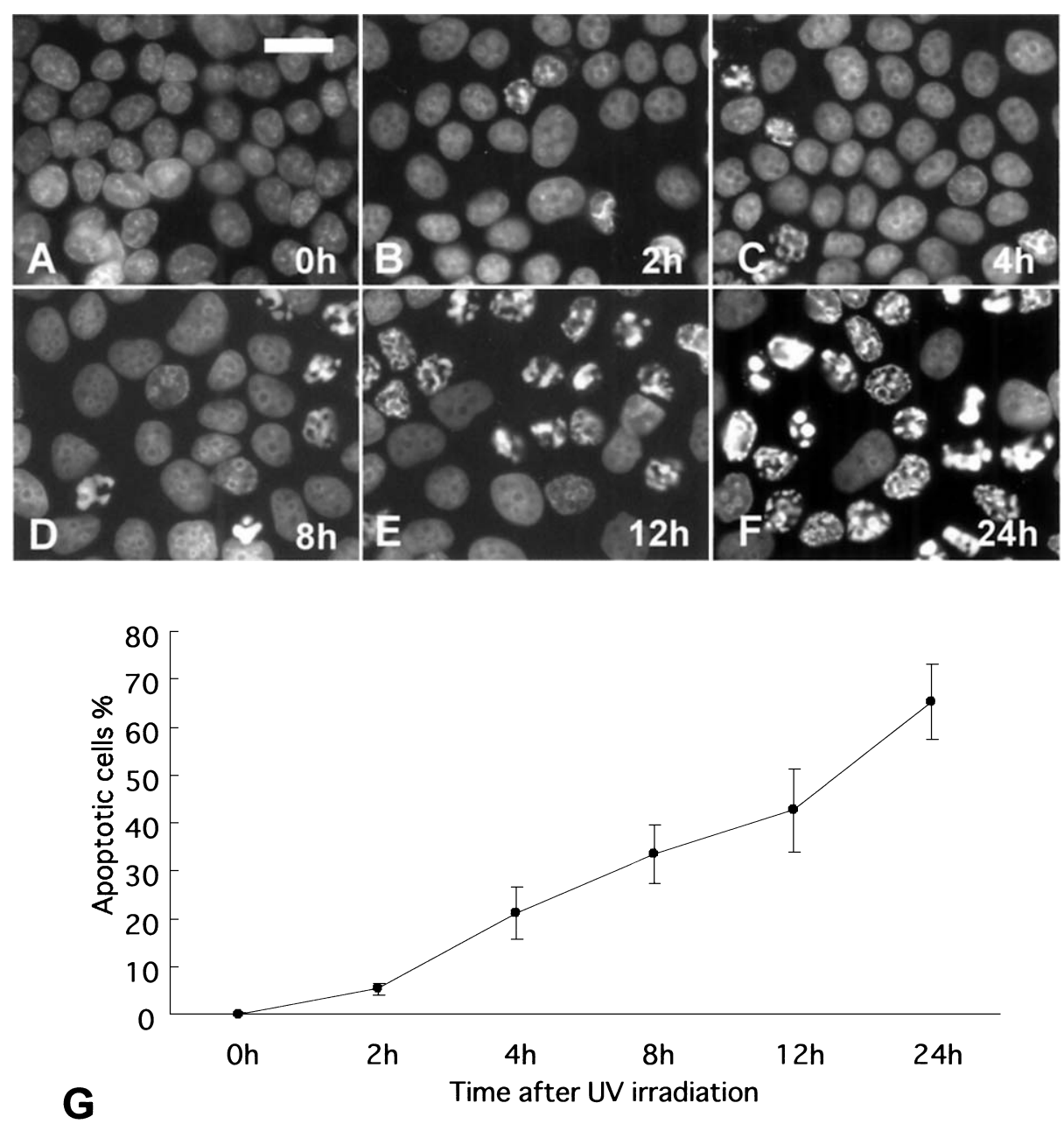

Fig. 2. Nuclear morphology of Cx43-EGFP-expressing HeLa cells after UV irradiation. Cells were harvested at the time-points indicated, then fixed with $4 \%$ paraformaldehyde, stained with Hoechst 33342, and examined by fluorescence microscopy (A-F). Cells with highly condensed chromatin or fragmented nuclei are visualized from $2 \mathrm{hr}$ after UV irradiation (B-F). Bar=30 $\mu \mathrm{m}$. The percentage (mean \pm S.D.) of cells with fragmented nuclei increases with the duration of post-irradiation culture $(\mathbf{G})$.

$94.2 \% \pm 3.6 \%$ at 2,4 , and $8 \mathrm{hr}$, respectively. However, with treatment with Z-VAD-FMK, the percentages decreased to $0 \%, 15.7 \% \pm 5.8 \%$ and $36.4 \% \pm 12.1 \%$ at 2,4 and $8 \mathrm{hr}$, respectively (Fig. 6E). These results indicated that the caspase inhibitor decreased the number of cells with MMP depolarization but could not completely inhibit cell death. Linear localization of Cx43-EGFP between cells was found at $2 \mathrm{hr}$ after UV and was preserved at $4 \mathrm{hr}$. Eight hours after UV irradiation with the $\mathrm{Z}-\mathrm{VAD}-\mathrm{FMK}$ treatment, although $\mathrm{Cx} 43$ EGFP plaques were observed at the areas where the MMP was lost, many of the strands of plaques were disorganized (Fig. 6D). The percentages of linear plaques with length of more than $5 \mu \mathrm{m}$ were calculated from 10 images at each time-point after UV irradiation with or without Z-VADFMK treatment (Fig. 6F). The percentages of linear plaques (cells with linear plaques/cells with Cx43-EGFP) were decreased after UV irradiation. Before irradiation, Cx43-EGFP showed conspicuous linear plaque localization (92\%). After UV irradiation without Z-VAD-FMK, the percentage of lin- ear plaques was reduced to $78.4 \%$ at $2 \mathrm{hr}$, and then declined sharply to $16.7 \%$ at $4 \mathrm{hr}$. At $8 \mathrm{hr}, \mathrm{Cx} 43$-EGFP linear plaques could hardly be detected. With the Z-VAD-FMK treatment, the percentages of linear plaques were $89.1 \%, 78.9 \%$ and $26.2 \%$ at 2,4 , and $8 \mathrm{hr}$, respectively. These observations demonstrated that Z-VAD-FMK could partly protect $\mathrm{Cx} 43$ EGFP and postpone its disorganization and degradation, suggesting that caspase is involved in the alteration of $\mathrm{Cx} 43$ EGFP.

\section{Discussion}

This study shows that, during apoptosis induced by UV irradiation, alteration of gap junctional plaques occurs after mitochondrial membrane potential depolarization but before nuclear fragmentation, and that this alteration depends partly on caspase activation. Using Cx43-EGFP-expressing HeLa cells, we found that intact Cx43-EGFP linear plaques were preserved not only among TMRE-positive cells but also 

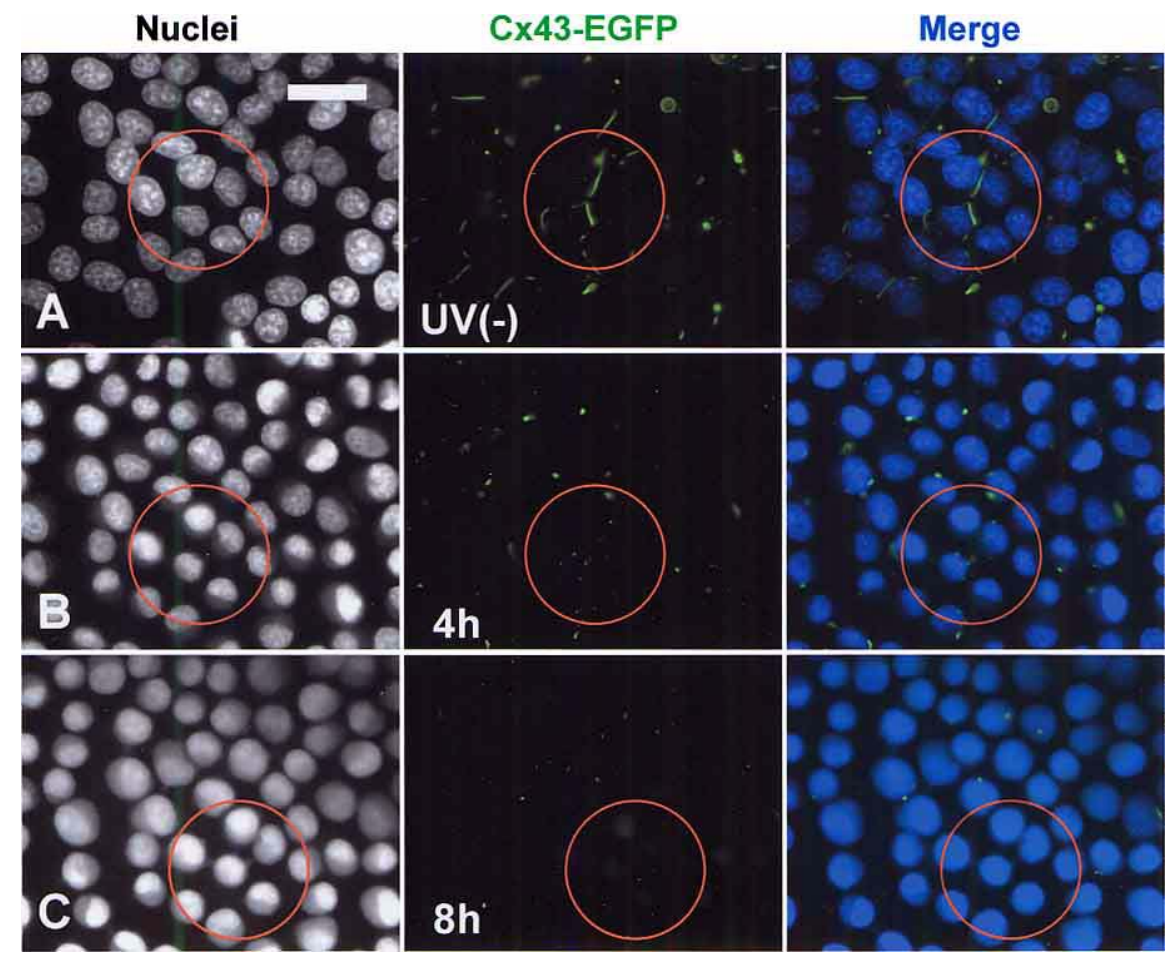

Fig. 3. Time-lapse observation of Cx43-EGFP-expressing HeLa cells after UV irradiation. Cx43-EGFP-expressing HeLa cells were stained with Hoechst 33342 and irradiated by UV-C. Time-lapse images of nuclei and Cx43-EGFP were taken at the time-point indicated in each row. Row $\mathbf{A}$ is the images of the field taken before UV irradiation, rows $\mathbf{B}$ and $\mathbf{C}$ are images of the same field at 4 and $8 \mathrm{hr}$, respectively, after UV treatment. Cx43-EGFP is disorganized into small dot-like pieces at $4 \mathrm{hr}(\mathbf{B})$ and is degraded at $8 \mathrm{hr}(\mathbf{C})$. The alteration of Cx43-EGFP occurs before changes of nuclear morphology. These images were pseudo-colored and merged by IPLab software. The red circles indicate an area containing the same cells. Bar $=30 \mu \mathrm{m}$.

among TMRE-negative cells at $2 \mathrm{hr}$ after UV irradiation; that small punctate of Cx43-EGFP were present in the cytoplasm at $4 \mathrm{hr}$ post-irradiation; and that Cx43-EGFP was diffusely localized in the cytoplasm at $8 \mathrm{hr}$ post-irradiation. We also demonstrated that treatment with the caspase inhibitor Z-VAD-FMK partially preserved Cx43-EGFP plaques and MMP at 4 and $8 \mathrm{hr}$ post-irradiation.

MMP depolarization is considered to be one of the early markers of apoptosis [3, 6]. During apoptosis induced by UV irradiation, we compared the time course of Cx43-EGFP plaque disorganization with MMP depolarization by TMRE staining. We noticed that even after cells lost their MMP, many cells still had intact Cx43-EGFP linear plaques at $2 \mathrm{hr}$ after UV irradiation (Fig. 4B). In other words, among the cells which had Cx43-EGFP linear plaques, about $40 \%$ of the cells had lost MMP (Fig. 4E). Furthermore, when cells showed diffuse localization of Cx43-EGFP in the cytoplasm at $8 \mathrm{hr}$ post-irradiation, all these cells had negative staining for TMRE (Fig. 4D). These results suggest that Cx43-EGFP is disorganized after MMP depolarization.

Cx43-EGFP was translocated to the cytoplasm before nuclear fragmentation. Nuclear fragmentation is regarded as a later marker of apoptosis. Time-lapse observation with Hoechst 33342 staining and fluorescence microscopy revealed that alteration of Cx43-EGFP localization occurred before nuclear fragmentation appeared (Figs. 3, 5C). In cells with condensed nuclei at $8 \mathrm{hr}$ post-irradiation, the $\mathrm{Cx} 43-$ EGFP in the cytoplasm was very weak (Fig. 5D). This observation suggested that Cx43-EGFP is degraded before nuclear fragmentation.

Fig. 4. Confocal laser scanning photomicrographs of Cx43-EGFP and TMRE after UV irradiation. Cells were stained with TMRE at the timepoints after UV irradiation indicated. The disorganization of Cx43-EGFP, depolarization of MMP, and cell morphology are observed after irradiation. Prominent linear Cx43-EGFP is found at the plasma membrane of cells without UV irradiation (row A); 2 hr after irradiation, the fluorescence intensity of TMRE is decreased and many cells have lost MMP; Cx43-EGFP plaques are linear and localized at the border of adjacent cells which show negative staining for TMRE (row B). At $4 \mathrm{hr}$, most cells have lost MMP, Cx43-EGFP is internalized into the cytoplasm, and many cells show diffuse localization of Cx43-EGFP in the cytoplasm (row C). At $8 \mathrm{hr}$, Cx43-EGFP has been degraded in the cytoplasm (row D). Bar=10 $\mu \mathrm{m}$. Quantitative analysis of TMRE staining at $2 \mathrm{hr}$ after UV irradiation shows that among the cells with preserved linear Cx43-EGFP plaques, 47.2\% show decreased TMRE fluorescence intensity and 39.6\% have lost MMP and show negative staining for TMRE (E). 
Cx43-EGFP

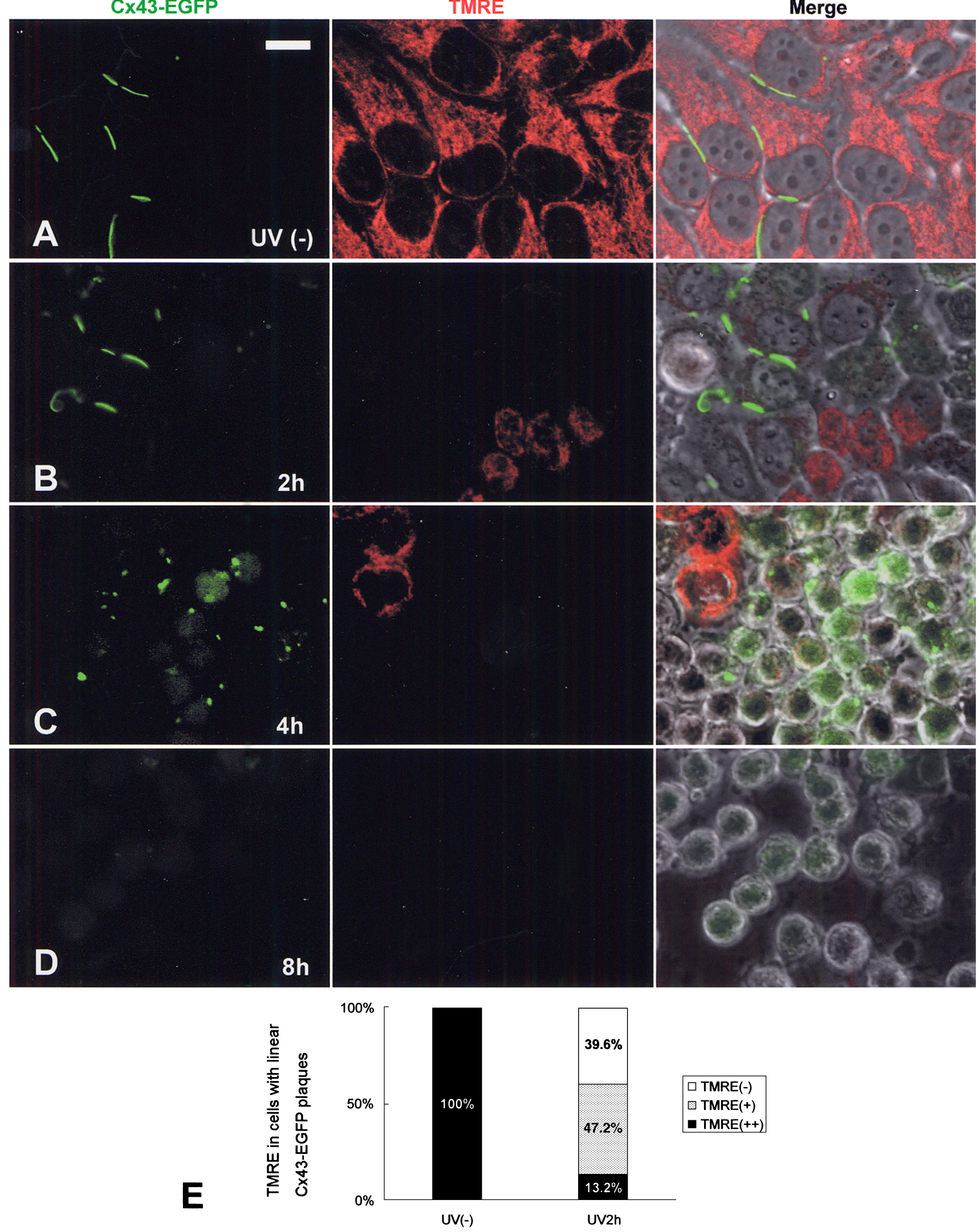

Fig. 4 

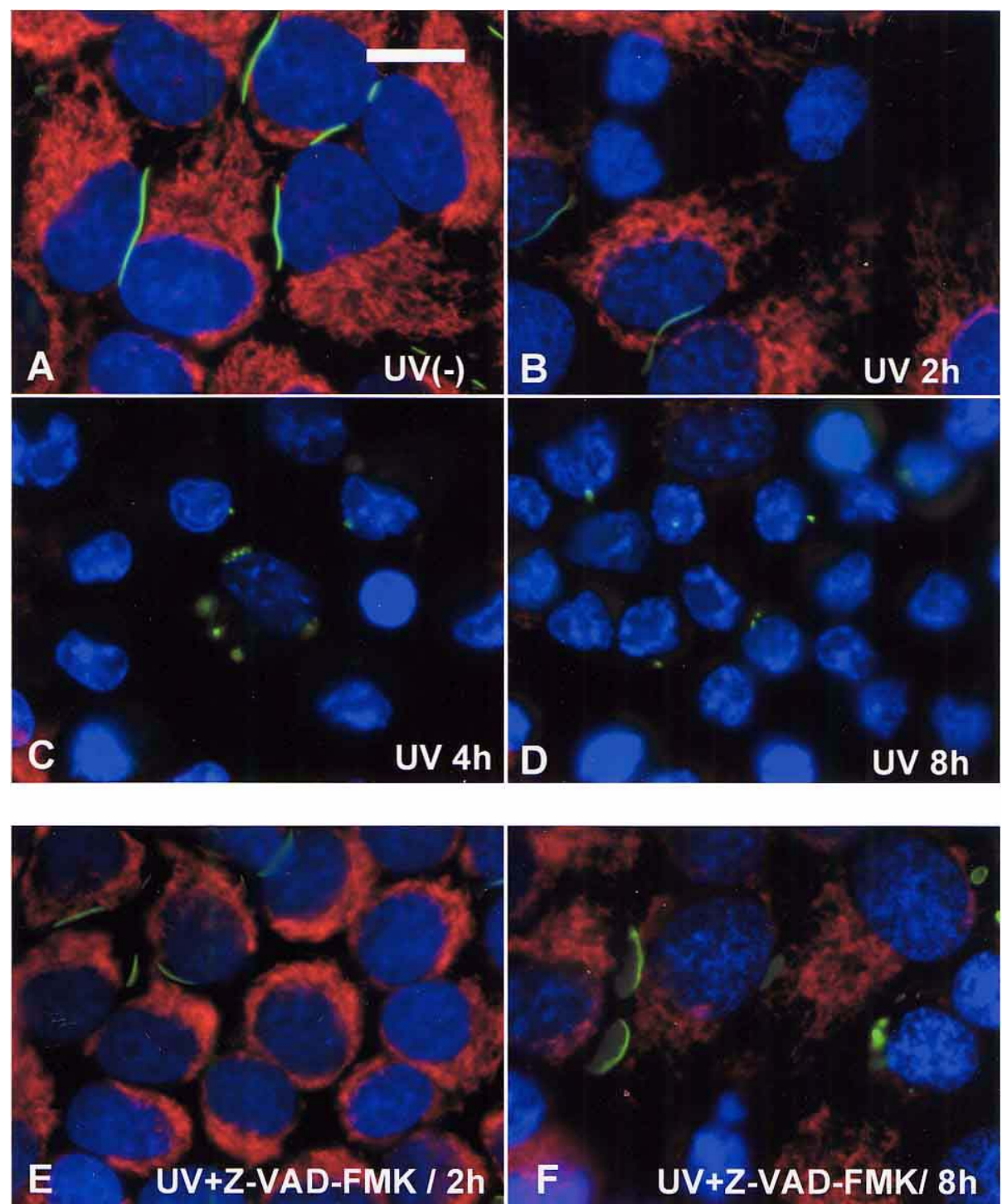

Fig. 5. Fluorescence microscopy images of cells treated or untreated with caspase inhibitor (Z-VAD-FMK) after UV irradiation. In UV (-) control experiment (A), all cells show positive staining for TMRE, and normal pattern of nuclei. The MMP of some cells has dissipated at $2 \mathrm{hr}$ after UV irradiation, but Cx43-EGFP plaques are preserved (B). At $4 \mathrm{hr}$, most cells show negative staining for TMRE, and Cx43-EGFP plaques are disorganized into small dots (C). At $8 \mathrm{hr}$, cells have lost MMP, and Cx43-EGFP is degraded into small dots; nuclear condensation is noticeable (D). With Z-VAD-FMK treatment, MMP depolarization is obviously inhibited at $2 \mathrm{hr}(\mathbf{E})$ and reduced at $8 \mathrm{hr}(\mathbf{F})$. Nuclear condensation is also inhibited at $8 \mathrm{hr}$ and linear Cx43-EGFP plaques and Cx43-EGFP dots can be seen (F). Cx43-EGFP, TMRE, and nuclei are pseudo-colored to green, red, and blue, respectively, and merged by IPLab software. Bar $=15 \mu \mathrm{m}$.

We can conceive of at least three possible mechanisms by which the $\mathrm{Cx} 43$ on the plasma membrane declines and is degraded during UV-induced apoptosis. Firstly, UV irradia- tion might lead to a decrease in connexin gene expression. $\mathrm{Cx} 43$ is a short-lived protein with a half-life of only a few hours $[13,17]$, and $\mathrm{Cx} 43$ plaques display dynamic structure.

Fig. 6. Confocal laser scanning microscopy images of cells treated with caspase inhibitor Z-VAD-FMK after UV irradiation. As compared with cells without caspase inhibitor treatment, MMP depolarization is blocked at $2 \mathrm{hr}$ (row B) and reduced at $4 \mathrm{hr}$ and $8 \mathrm{hr}$ (rows $\mathbf{C}$ and D). Morphometric analysis of TMRE staining shows that $33.7 \% \pm 6.0 \%, 78.1 \% \pm 12.0 \%$, and $94.2 \% \pm 3.6 \%$ of cells have lost MMP at 2,4 , and $8 \mathrm{hr}$, respectively; however, with the Z-VAD-FMK treatment, the percentages declined to $0 \%, 15.7 \% \pm 5.8 \%$, and $36.4 \% \pm 12.1 \%$, respectively. The means and standard deviations for the percentages from ten pictures at each time-point are shown (E). Morphometric analysis of Cx43-EGFP demonstrates that linear Cx43-EGFP plaques are conspicuous at $2 \mathrm{hr}$ with or without Z-VAD-FMK treatment, and that they are preserved at $4 \mathrm{hr}$ with the treatment. Eight hours after UV and Z-VAD-FMK treatment, Cx43-EGFP plaques are observed; however, many plaques are disorganized (D). Bar=10 $\mu \mathrm{m}$. Disorganization of Cx43-EGFP is postponed by the caspase inhibitor $(\mathbf{F})$. 
Cx43-EGFP
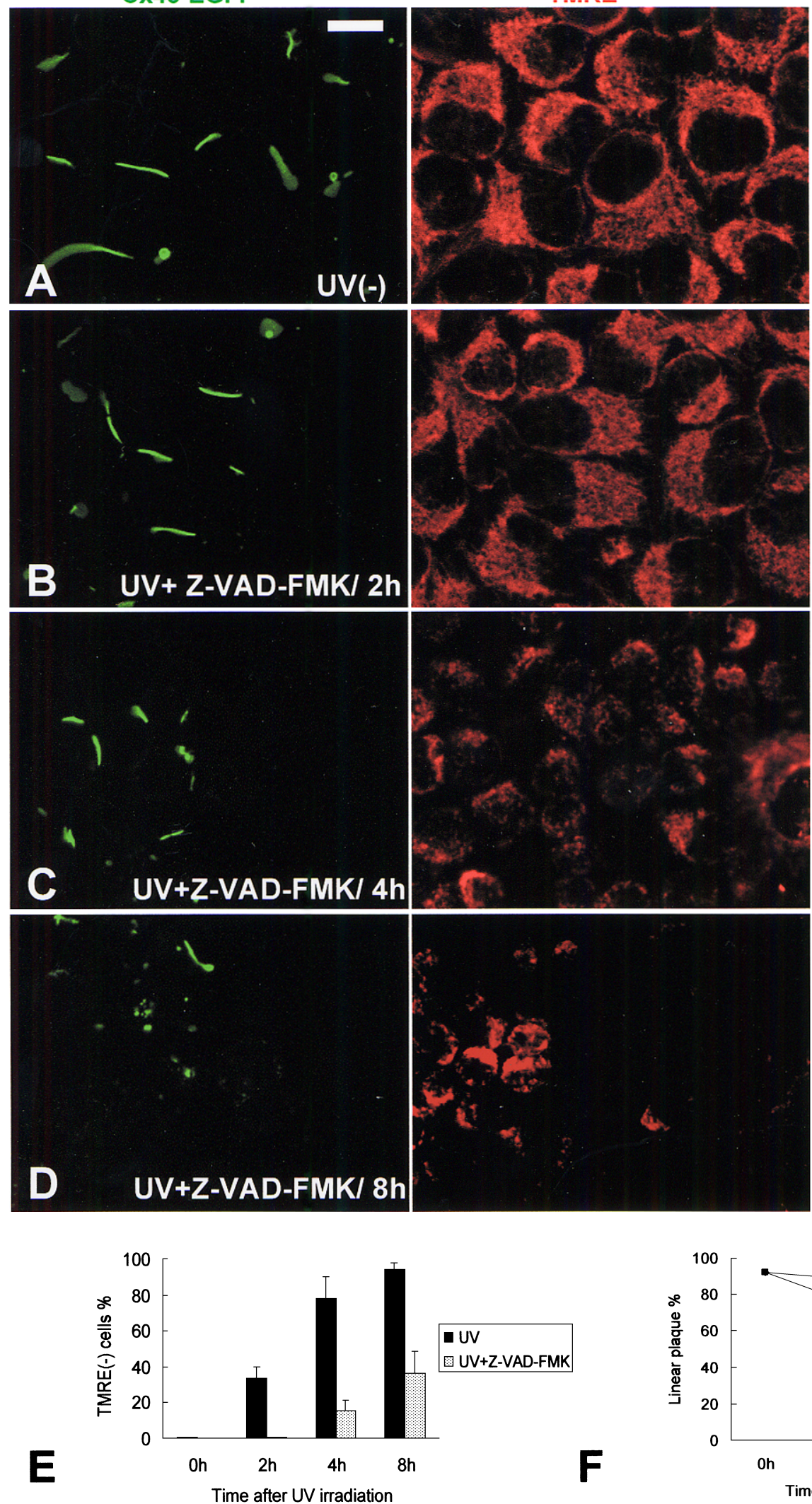

Merge
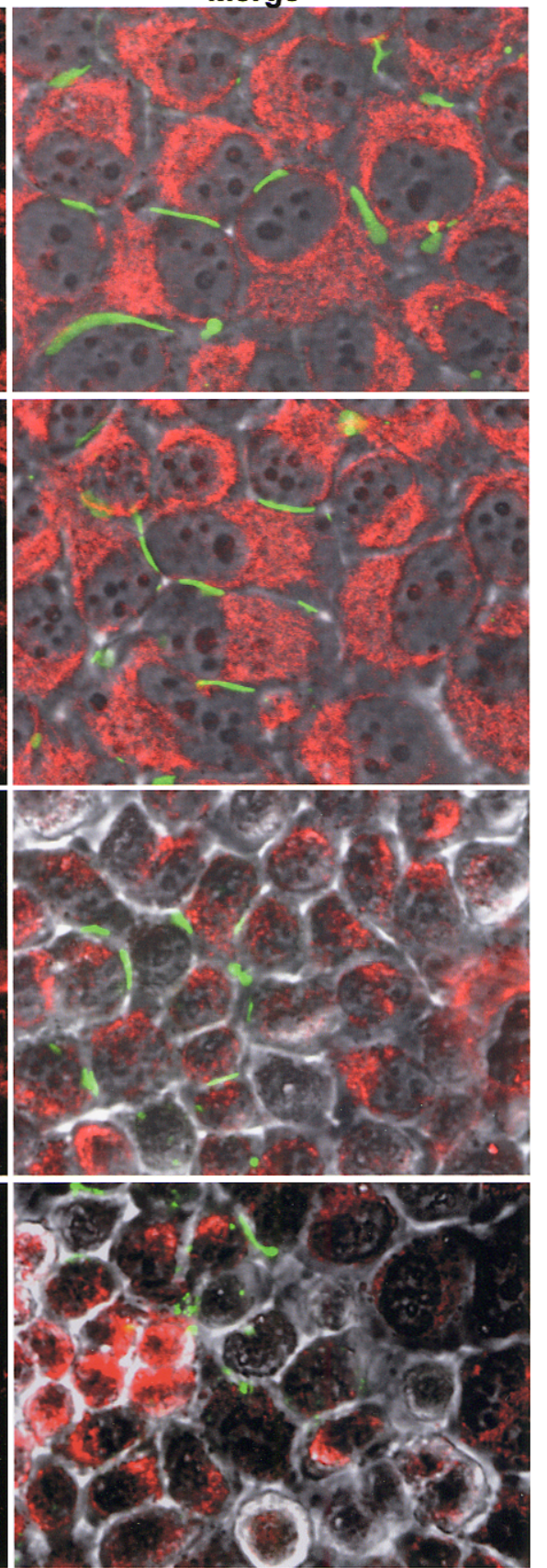

F

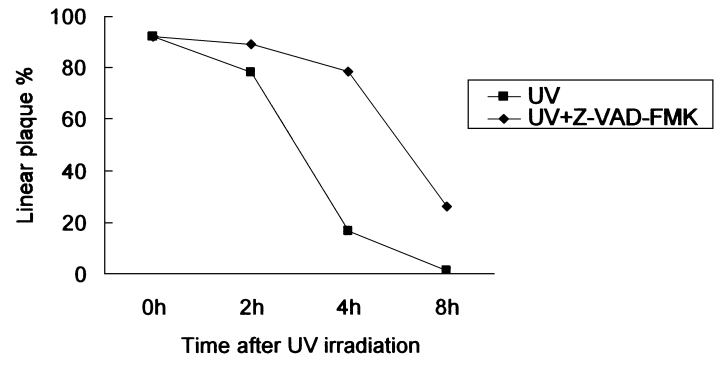

Fig. 6 
Newly synthesized $\mathrm{Cx} 43$ connexons are delivered in vesicular carriers traveling along microtubules from the Golgi apparatus to the plasma membrane and accumulate in plaques [14]. Synthesis and degradation of $\mathrm{Cx} 43$ plaques are very dynamic processes. The expression of $\mathrm{Cx} 43$ is subject to a variety of stresses. It was reported that low dose of UV-C irradiation on WB-F344 cells resulted in significant reduction in levels of the $\mathrm{Cx} 43$ native isoform, although no apparent changes in the phosphorylated form were detected [1]. However, the effect of UV-C irradiation on expression of Cx43 appears to be cell-type specific; in AG1522 cells, no decrease in Cx43 levels was observed [1].

Secondly, lysosomes and proteasomes could be involved in $\mathrm{Cx} 43$ degradation during UV-induced apoptosis. Lysosomes and proteasomes play different roles in the life cycle of $\mathrm{Cx} 43$. Active proteasomal degradation was found to reduce the amount of phosphorylated $\mathrm{Cx} 43$, and to destabilize the gap junctions at the plasma membrane. Internalized $\mathrm{Cx} 43$ is targeted by lysosomes for degradation [12, 22].

Thirdly, under apoptosis-inducing stimuli, connexins might be a substrate of caspase protease. For example, Cx45.6 could be regulated by apoptotic protease caspase- 3 and cleaved by caspase-3-like protease [26]. Cx45.6 was the first connexin identified as a substrate for caspase. Our observation indicated that caspase partly contributes to the degradation of $\mathrm{Cx} 43$. In our study, Z-VAD-FMK, a broadspectrum caspase inhibitor, inhibited MMP depolarization and blocked nuclear fragmentation. Interestingly, Z-VADFMK could also postpone the disorganization of $\mathrm{Cx} 43$ EGFP, i.e., Cx43-EGFP linear plaques were still predominant $(78.9 \%)$ at $4 \mathrm{hr}$ after UV irradiation. However, $\mathrm{Cx} 43-$ EGFP was disorganized at $8 \mathrm{hr}$. The percentage of linear plaques was decreased to $26.2 \%$ at $8 \mathrm{hr}$ after irradiation with Z-VAD-FMK treatment (Fig. 6F). On the other hand, in the cells without Z-VAD-FMK treatment, Cx43-EGFP linear plaques decreased in number at $4 \mathrm{hr}(16.7 \%)$ and could hardly be observed at $8 \mathrm{hr}$ (Figs. 4C, 4D, 5C, 5D, 6F). These results suggest that caspase activation partially contributes to $\mathrm{Cx} 43$ alteration during apoptosis induced by UV irradiation. On the other hand, we cannot completely rule out the possibility that the disorganization of gap junction plaques is a common phenomenon occurring during cell death including apoptosis and necrosis, since Z-VAD-FMK significantly inhibited UV-induced MMP depolarization and disorganization of gap junction plaques but could not completely inhibit them. In other words, even in the presence of a caspase inhibitor, the cell death processes probably other than apoptosis, such as necrosis, may occur in a number of cells in which cell gap junctions are disorganized.

In conclusion, this study showed that during apoptosis induced by UV irradiation, disorganization of gap junction plaques occurs after MMP depolarization but before nuclear fragmentation, and that this alteration partly depends on caspase activation.

\section{Acknowledgments}

This work was supported in part by grants-in-aid for scientific research from the Ministry of Education, Culture, Sports, Science, and Technology, Japan.

We thank Ramona Ratliff for advice on manuscript preparation.

\section{References}

1. Azzam, E. I., de Toledo, S. M. and Little, J. B. (2003) Expression of connexin 43 is highly sensitive to ionizing radiation and other environmental stresses. Cancer Res. 63; 7128-7135.

2. Beyer, E. C., Paul, D. L. and Goodenough, D. A. (1990) Connexin family of gap junction proteins. J. Membr. Biol. 116; 187-194.

3. Blom, W. M., de Bont, H. J. and Nagelkerke, J. F. (2003) Regional loss of the mitochondrial membrane potential in the hepatocyte is rapidly followed by externalization of phosphatidylserines at that specific site during apoptosis. J. Biol. Chem. 278; 1246712474.

4. Bruzzone, R. and Ressot, C. (1997) Connexins, gap junctions and cell-cell signalling in the nervous system. Eur. J. Neurosci. 9; 16.

5. Dunkern, T. R., Fritz, G. and Kaina, B. (2001) Ultraviolet lightinduced DNA damage triggers apoptosis in nucleotide excision repair-deficient cells via $\mathrm{Bcl}-2$ decline and caspase-3/-8 activation. Oncogene 20; 6026-6038.

6. Goldstein, J. C., Waterhouse, N. J., Juin, P., Evan, G. I. and Green, D. R. (2000) The coordinate release of cytochrome c during apoptosis is rapid, complete and kinetically invariant. Nat. Cell Biol. 2; 156-162.

7. Goodenough, D. A., Goliger, J. A. and Paul, D. L. (1996) Connexins, connexons, and intercellular communication. Annu. Rev. Biochem. 65; 475-502.

8. Huang, R. P., Hossain, M. Z., Huang, R., Gano, J., Fan, Y. and Boynton, A. L. (2001) Connexin 43 (Cx43) enhances chemotherapy-induced apoptosis in human glioblastoma cells. Int. J. Cancer 92; 130-138.

9. Krutovskikh, V. A., Piccoli, C., Yamasaki, H. and Yamasaki, H. (2002) Gap junction intercellular communication propagates cell death in cancerous cells. Oncogene 21; 1989-1999.

10. Kumar, N. M. and Gilula, N. B. (1996) The gap junction communication channel. Cell 84; 381-388.

11. Kumar, S. (1999) Mechanisms mediating caspase activation in cell death. Cell Death Differ. 6; 1060-1066.

12. Laing, J. G., Tadros, P. N., Westphale, E. M. and Beyer, E. C. (1997) Degradation of connexin43 gap junctions involves both the proteasome and the lysosome. Exp. Cell Res. 236; 482-492.

13. Laird, D. W., Puranam, K. L. and Revel, J. P. (1991) Turnover and phosphorylation dynamics of connexin 43 gap junction protein in cultured cardiac myocytes. Biochem. J. 273; 67-72.

14. Lauf, U., Giepmans, B. N., Lopez, P., Braconnot, S., Chen, S. C. and Falk, M. M. (2002) Dynamic trafficking and delivery of connexons to the plasma membrane and accretion to gap junctions in living cells. Proc. Natl. Acad. Sci. U S A 99; 10446-10451.

15. Lin, J. H., Yang, J., Liu, S., Takano, T., Wang, X., Gao, Q., Willecke, K. and Nedergaard, M. (2003) Connexin mediates gap junction-independent resistance to cellular injury. J. Neurosci. 23; 430-441.

16. Mills, J. C., Stone, N. L. and Pittman, R. N. (1999) Extranuclear apoptosis, the role of the cytoplasm in the execution phase. J. Cell Biol. 146; 703-708.

17. Musil, L. S., Le, A. C., VanSlyke, J. K. and Roberts, L. M. (2000) Regulation of connexin degradation as a mechanism to increase gap junction assembly and function. J. Biol. Chem. 275; 25207- 
25215.

18. Nicholson, D. W. (1999) Caspase structure, proteolytic substrates, and function during apoptotic cell death. Cell Death Differ. 6; 1028-1042.

19. Oyamada, Y., Zhou, W., Oyamada, H., Takamatsu, T. and Oyamada M. (2002) Dominant-negative connexin43-EGFP inhibits calcium-transient synchronization of primary neonatal rat cardiomyocytes. Exp. Cell Res. 273; 85-94.

20. Paul, D. L. (1995) New functions for gap junctions. Curr. Opin. Cell Biol. 7; 665-672.

21. Plotkin, L. I., Manolagas, S. C. and Bellido, T. (2002) Transduction of cell survival signals by connexin- 43 hemichannels. J. Biol. Chem. 277; 8648-8657.

22. Qin, H., Shao, Q., Igdoura, S. A., Alaoui-Jamali, M. A. and Laird, D. W. (2003) Lysosomal and proteasomal degradation play distinct roles in the life cycle of $\mathrm{Cx} 43$ in gap junctional intercellular communication-deficient and -competent breast tumor cells. $J$. Biol. Chem. 278; 30005-30014.

23. Sasai, K., Yajima, H. and Suzuki, F. (2002) Suppression of postmitochondrial signaling and delayed response to UV-induced nuclear apoptosis in HeLa cells. Jpn. J. Cancer Res. 93; 275-283.

24. Sohl, G. and Willecke, K. (2003) An update on connexin genes and their nomenclature in mouse and man. Cell Commun. Adhes. $10 ; 173-180$.

25. Wilson, M. R., Close, T. W. and Trosko, J. E. (2000) Cell population dynamics (apoptosis, mitosis, and cell-cell communication) during disruption of homeostasis. Exp. Cell Res. 254; 257-268.

26. Yin, X., Gu, S. and Jiang, J. X. (2001) The development-associated cleavage of lens connexin 45.6 by caspase-3-like protease is regulated by casein kinase II-mediated phosphorylation. J. Biol. Chem. 276; 34567-34572. 